\title{
Some aspects of the development of sex differences in DRL behavior
}

\author{
WILLIAM W. BEATTY, DANIEL R. STUDELSKA, and JEFFREY M. GERTH \\ North Dakota State University, Fargo, North Dakota 58102
}

\begin{abstract}
Adult female rats acquired efficient DRL performance more rapidly than males. This sex difference was not observed in rats that began training as weanlings because both young males and females acquired efficient DRL performance about as rapidly as adult females and more rapidly than adult males. Although it is possible that proficiency on the DRL task declines with age in males, differences in the effortfulness of the response or the incentive value of the reward may explain the observed age difference.
\end{abstract}

When trained in adulthood, female rats generally acquire efficient DRL performance more rapidly than males (Beatty, 1973; Kearley, van Hartesveldt, \& Woodruff, 1974). Activational effects of ovarian hormones are at least partially responsible for this sex difference in performance (Beatty, 1973). The initial experiment was designed to examine the ontogeny of sex differences in this behavior. Open-field behavior was also studied because the ontogeny of sex differences in this behavior has been established (Beatty \& Fessler, in press).

\section{EXPERIMENT I}

\begin{abstract}
Method
Subjects. The animals were 8 female and 12 male albino rats born in the laboratory from pregnant dams purchased from the Holtzman Company, Madison, Wisconsin. The animals were reared by their natural mothers until weaning at 21 days of age, when they were weighed, placed in single cages, and fed an amount necessary to maintain body weight at $85 \%$ of that of an equal number of littermates given food ad lib. Once daily, on Days 22-25, the animals received 3-min tests in an open field, as described elsewhere (Beatty \& Fessler, in press). On Day 26 the rats were shaped to barpress for $20-\mathrm{mg}$ Noyes pellets in standard Gerbrands chambers. On Days 27-28 they were trained on CRF until they earned at least 60 reinforcements. Beginning at Day 65 , the rats were retested in the open field for 4 consecutive days under free-feeding conditions.
\end{abstract}

\section{Results}

As seen in Table 1, there were no differences between young males and females in any aspect of DRL performance $(\mathrm{F}<1$ for responses and reinforcements, $\mathrm{F}=$ 1.52 for efficiency). Both sexes acquired efficient DRL performance about as rapidly as adult females tested in earlier experiments (Beatty, 1973) and considerably more rapidly than adult males. There were no sex differences in open-field behavior in the first test, but in the second test at 65 days of age females were more active and reared more frequently (both ps $<.01$ ), replicating earlier results (Beatty \& Fessler, in press).

This research was supported by PHS Grant HD 08206 from NICHHD.
Table 1

Mean Performance in Experiment $I$

\begin{tabular}{lcrrrrrr}
\hline & \multicolumn{7}{c}{ Blocks of Five Sessions } \\
& 1 & 2 & 3 & 4 & 5 & 6 \\
\hline $\begin{array}{l}\text { Responses } \\
\quad \text { Males }\end{array}$ & 171.2 & 133.8 & 107.5 & 95.0 & 91.4 & 89.7 \\
$\quad \begin{array}{l}\text { Females } \\
\text { Reinforcements }\end{array}$ & 149.4 & 122.4 & 106.5 & 101.8 & 94.7 & 94.1 \\
$\quad$ Males & 12.4 & 18.9 & 31.2 & 41.0 & 40.3 & 42.2 \\
$\quad \begin{array}{l}\text { Females } \\
\text { Efficiency }\end{array}$ & 15.6 & 22.3 & 32.0 & 37.9 & 41.9 & 45.9 \\
$\quad$ Males & 19.8 & 22.6 & 29.9 & 34.7 & 34.8 & 35.3 \\
$\quad$ Females & 22.4 & 25.5 & 31.5 & 35.7 & 37.9 & 41.3 \\
\hline
\end{tabular}

\section{EXPERIMENT II}

The rapid acquisition of DRL behavior by young rats of both sexes in light of the much poorer performance by adult males in earlier studies was surprising considering what is known of the ontogeny of activity (e.g., Moorcroft, Lytle, \& Campbell, 1971) and passive avoidance behavior (Riccio, Rohrbaugh, \& Hodges, 1968; Schulenburg, Riccio, \& Stikes, 1971) in the rat. We suspected that the apparently better performance by younger males might reflect some procedural difference between the earlier study with adults (Beatty, 1973) and Experiment I. One such difference was in the method of deprivation. In the earlier study animals were maintained throughout DRL training at $85 \%$ of their ad-lib body weight levels prior to beginning training, while in Experiment I the animals were maintained at $85 \%$ of the body weight of free-fed controls of the same sex. The second experiment compared the effects of these two deprivation methods on the DRL behavior of adult rats.

\section{Method}

Subjects. The animals were adult Holtzman rats that had served in an active avoidance experiment using procedures that have been described elsewhere (Beatty \& Beatty, 1970) between 105 and 115 days of age. Beginning at about 125 days of age, baseline body weights were established under freefeeding conditions and the rats were reduced to $85 \%$ of this 
Table 2

Mean Performance in Experiment II

\begin{tabular}{lrrrrrr}
\hline & \multicolumn{7}{c}{ Blocks of Five Sessions } \\
& 1 & 2 & 3 & \multicolumn{1}{c}{4} & \multicolumn{1}{c}{5} & \multicolumn{1}{c}{6} \\
\hline \multicolumn{1}{c}{ Responses } & & & & & & \\
Male-Adjusted & 199.6 & 173.1 & 144.7 & 134.1 & 129.8 & 123.8 \\
Female-Adjusted & 199.1 & 171.4 & 139.4 & 118.4 & 97.2 & 86.6 \\
Male-Constant & 184.8 & 168.5 & 148.6 & 154.7 & 138.0 & 133.2 \\
Female-Constant & 199.5 & 180.9 & 145.9 & 133.1 & 118.9 & 108.9 \\
Reinforcements & & & & & & \\
Male-Adjusted & 19.0 & 15.9 & 16.3 & 19.1 & 22.7 & 27.5 \\
Female-Adjusted & 18.3 & 16.1 & 22.3 & 31.5 & 40.6 & 45.7 \\
Male-Constant & 18.6 & 13.8 & 15.0 & 12.9 & 19.7 & 24.8 \\
Female-Constant & 17.4 & 16.1 & 23.1 & 30.2 & 36.9 & 42.1 \\
$\quad$ Efficiency & & & & & & \\
Male-Adjusted & 11.4 & 9.3 & 11.5 & 15.5 & 19.1 & 26.7 \\
Female-Adjusted & 9.8 & 12.9 & 20.9 & 33.5 & 46.6 & 56.4 \\
Male-Constant & 11.0 & 8.8 & 11.2 & 9.2 & 17.1 & 24.5 \\
Female-Constant & 9.6 & 11.0 & 20.1 & 29.2 & 37.8 & 43.8 \\
\hline
\end{tabular}

weight. The animals were randomly assigned to groups that were maintained at $85 \%$ of the baseline weight level (i.e., constant weight groups, $N=12$ males and 13 females) or allowed to gain weight at $85 \%$ of the rate of a group of males and females of identical age and past experience that were fed ad lib (i.e., adjusted weight groups, $N=13$ males and 13 females). At 140 days of age, the rats were shaped to barpress for $45-\mathrm{mg}$ Noyes pellets in a single session, given one additional session where they earned reinforcements on CRF, and tested for 30 daily 30-min sessions on DRL 20.

\section{Results}

As seen in Table 2, female rats earned more reinforcements and performed more efficiently $(F s=14.55$ and 17.30, respectively, df $=1 / 47$, ps $<.001)$. Sex differences on these measures were not apparent early in training but developed during the later sessions (Sex by Blocks: $F s=13.03$ and 12.62 for reinforcements and efficiency, respectively, $\mathrm{df}=5 / 235$, ps $<.001$ ). Although the main effect of sex was not significant in the analysis of total responses, there was a reliable interaction of Sex by Blocks $(F=5.78, \mathrm{df}=5 / 235$, $\mathrm{p}<.001$ ), reflecting the lower levels of responding by female groups during the later stages of training. The type of deprivation procedure did not significantly affect performance on any measure, nor did the method of deprivation interact with the sex of the subject.

\section{DISCUSSION}

Regardless of whether or not the deprivation schedule corrected for growth, adult female rats acquired efficient DRL performance more rapidly than males, in agreement with earlier findings (Beatty, 1973; Kearley et al., 1974). In younger animals no such sex difference was observed, principally because of the unexpectedly competent performance of the young males. While it is possible that the capacity for acquiring efficient DRL performance deteriorates with age in male but not in female rats, this seems unlikely considering the fact that young rats acquire passive avoidance responses more slowly than adults (Riccio et al., 1968; Schulenburg et al., 1971) and other simple behaviors at about the same rate as older animals (Campbell, 1967).
Instead, the apparent age difference may reflect one of many differences in procedure that necessarily occurred in the tests of younger and older animals. First, differences in deprivation cannot be ruled out as contributing factors, although the failure to find a significant influence of deprivation method in the second experiment suggests this factor is not especially important. Moreover, other data (Conrad, Sidman, \& Herrnstein, 1968; Holz \& Azrin, 1963) suggest that varying deprivation has little effect on DRL performance unless very mild deprivation is used.

Differences in the magnitude of incentive available to the young and old rats may be a more important factor in the age difference in DRL we observed. The younger rats were tested with $20-\mathrm{mg}$ pellets, while $45-\mathrm{mg}$ pellets were used with adults because previous research (Campbell, Jaynes, \& Misanin, 1968) suggested these rewards produce comparable rates of satiation in weanlings and adults. However, the month-long interval required for the DRL test may have resulted in an inequality in reward magnitude between the different age groups during the latter stages of training. Since reducing magnitude of reinforcement improves DRL efficiency (Beer \& Trumble, 1965), an effectively reduced magnitude of reward might explain the age difference in males. The absence of a comparable effect in females might simply reflect their lesser growth rate.

Finally, differences in the amount of effort required to make the barpress response might be important in the age difference in DRL behavior observed in males. We adjusted the force required to depress the bars to the minimum value consistent with reliable operation (approximately 17-19 $\mathrm{g}$ for the younger rats and set the bars so that $25 \mathrm{~g}$ were required to activate the mechanism for adults in an attempt to equate this variable. However, since the height of the bar above the grid floor was constant for both ages, the effective response effortfulness may have been greater for the younger rats, a possibility that is supported by their somewhat lower rates of responding during the initial sessions.

Obviously, the present data do not definitively establish an age difference in the DRL behavior in male rats. The relatively rapid maturation of this species combined with the lengthy testing necessary for acquisition of reasonably efficient performance may well preclude, or at least render impractical, a valid test of the ontogeny of this behavior in the rat. Nevertheless, the relatively proficient performance of the young animals in the present study suggests that cholinergic and other inhibitory mechanisms, probably in the forebrain, which are clearly important in controlling DRL behavior in the adult rat (see, e.g., Carlton, 1963, 1968; Ross \& Grossman, 1974 ), are sufficiently mature before 60 days of age to permit the development of efficient DRL performance.

\section{REFERENCES}

BEATTY, W. W. Effects of gonadectomy on sex differences in DRL behavior. Physiology and Behavior, 1973, 10, 177-178.

Beatty, W. W., \& Beatty, P. A. Hormonal determinants of sex differences in active avoidance behavior and reactivity to electric shock in the rat. Journal of Comparative and Physiological Psychology, 1970, 73, 446-455.

BeattY, W. W., \& Fessler, R. G. Ontogeny of sex differences in open-field behavior and sensitivity to electric shock in the rat. Physiology and Behavior, in press.

BeER, B., \& Trumble, G. Timing behavior as a function of amount of reinforcement. Psychonomic Science, 1965, 2, $71-72$.

Campbell, B. A. Developmental studies of learning and motivation in infraprimate mammals. In H. W. Stevenson, E. H. Hess, and H. L. Rheingold (Eds.), Early behavior: Comparative and developmental approaches. New York: Wiley, 1967. 
Campbell, B. A., Jaynes, J., \& Misanin, J. R. Retention of a light-dark discrimination in rats of different ages. Journal of Comparative and Physiological Psychology, $1968,66,467-472$.

Carlton, P. L. Cholinergic mechanisms in the control of behavior by the brain. Psychological Review, 1963, 70, 19-39.

Carlton, P. L. Cholinergic mechanisms in the control of behavior. In D. H. Efron (Ed.), Psychopharmacology: A review of progress 1957-1967. (USPHS Publ. Rep. No. 1836) Washington, D.C: U.S. Government Printing Office, 1968.

Conrad, D. G., Sidman, M., \& Herrnstein, R. J. The effect of deprivation upon temporally spaced responding. Journal of the Experimental Analysis of Behavior, 1958, $1,59-65$.

Holz, W. C., \& AzRin, N. H. A comparison of several procedures for eliminating behavior. Journal of the Experimental Analysis of Behavior, 1963, 6, 399-406.

KeARley, R. C., van Hartlesveldt, C., \& Woodruff, M. L. Behavioral and hormonal effects of hippocampal lesions on male and female rats. Physiological Psychology, $1974,2,187-196$.
Moorcroft, W. H., Lytle, L. D., \& Campbell, B. A. Ontogeny of starvation-induced behavioral arousal in the rat. Journal of Comparative and Physiological Psychology, 1971, 75, 59-67.

Riccio, D. C., Rohrbaugh, M., \& Hodges. L. A. Developmental aspects of passive and active avoidance learning in rats. Developmental Psychobiology, 1968, 1, 108-111. Ross, J. F., \& Grossman, S. P. Intrahippocampal application of cholinergic agents and blockers: Effects on rats in differential reinforcement of low rates and Sidman avoidance paradigms. Journal of Comparative and Physiological Psychology, 1974, 86, 590-600.

Schulenburg, C. J., Riccio, D. C., \& Stikes, E. R. Acquisition and retention of a passive-avoidance response as a function of age in rats. Journal of Comparative and Physiological Psychology, 1971, 74, 75-83.

(Received for publication September 15, 1975.) 